\title{
Fructose in the kidney: from physiology to pathology
}

\author{
Takahiko Nakagawa $^{1}$, Duk-Hee Kang ${ }^{2}$ \\ ${ }^{1}$ Department of Nephrology, Rakuwakai Otowa Hospital, Kyoto, Japan \\ ${ }^{2}$ Division of Nephrology, Department of Internal Medicine, Ewha Medical Research Institute, Ewha Womans University College of Medicine, \\ Seoul, Republic of Korea
}

The Warburg effect is a unique property of cancer cells, in which glycolysis is activated instead of mitochondrial respiration despite oxygen availability. However, recent studies found that the Warburg effect also mediates non-cancer disorders, including kidney disease. Currently, diabetes or glucose has been postulated to mediate the Warburg effect in the kidney, but it is of importance that the Warburg effect can be induced under nondiabetic conditions. Fructose is endogenously produced in several organs, including the kidney, under both physiological and pathological conditions. In the kidney, fructose is predominantly metabolized in the proximal tubules; under normal physiologic conditions, fructose is utilized as a substrate for gluconeogenesis and contributes to maintain systemic glucose concentration under starvation conditions. However, when present in excess, fructose likely becomes deleterious, possibly due in part to excessive uric acid, which is a by-product of fructose metabolism. A potential mechanism is that uric acid suppresses aconitase in the Krebs cycle and therefore reduces mitochondrial oxidation. Consequently, fructose favors glycolysis over mitochondrial respiration, a process that is similar to the Warburg effect in cancer cells. Activation of glycolysis also links to several side pathways, including the pentose phosphate pathway, hexosamine pathway, and lipid synthesis, to provide biosynthetic precursors as fuel for renal inflammation and fibrosis. We now hypothesize that fructose could be the mediator for the Warburg effect in the kidney and a potential mechanism for chronic kidney disease.

Keywords: Fructose, Glycolysis, Inflammation, Mitochondria, Proximal tubules, Uric acid, Warburg effect

\section{Introduction}

Fructose is a natural sugar present in fruits and honey and is a fundamental nutrient for wild animals. Bears, squirrels, birds migrating over long distances, and freshwater Pacu fish actively eat fruits to accumulate fat, presumably as a protection against periods of food shortage [1]. Fructose contributes to lipid and glycogen syntheses for energy stor- age, and to the development of insulin resistance to prevent glucose utilization in the peripheral tissue, and glucose delivery to the central nervous system. In addition, fructose also stimulates salt reabsorption to raise blood pressure (as discussed in the following section) [2]. Fructose is also metabolized under hypoxic conditions and often exhibits the protective effect. A recent study examined how the naked mole rat could survive long periods of hypoxic conditions

Received: July 6, 2021; Revised: August 10, 2021; Accepted: August 13, 2021

Editor: Tae-Hyun Yoo, Yonsei University, Seoul, Republic of Korea

Correspondence: Takahiko Nakagawa

Department of Nephrology, Rakuwakai Otowa Hospital, 2 Otowa-Chinji-cho, Yamashina-ku, Kyoto 607-8062, Japan.

E-mail:nakagawt@gmail.com

ORCID: https://orcid.org/0000-0002-0127-1529

Copyright (C) 2021 by The Korean Society of Nephrology

(a) This is an Open Access article distributed under the terms of the Creative Commons Attribution Non-Commercial and No Derivatives License (http:// creativecommons.org/licenses/by-nc-nd/4.0/) which permits unrestricted non-commercial use, distribution of the material without any modifications, and reproduction in any medium, provided the original works properly cited. 
and found that it was attributed to their ability to produce fructose endogenously in several organs, which is subsequently metabolized to provide several biosynthetic precursors required for cell survival, including nucleic acids, amino acids, lipids and energy [3]. Likewise, the reason why the fetus exposed to hypoxia can survive during early pregnancy is that the developing placenta also produces endogenous fructose, likely aiding fetal organ growth in wild animals as well as humans [4-6].

In modern society, fructose, as a component of high-fructose corn syrup or table sugar, is preferentially added to sugar-sweetened soft drinks and sodas. A dramatic increase in fructose consumption is, however, associated with a high prevalence of the metabolic syndrome, stimulating a heated debate over the potential danger of sugar-sweetened beverages (SSB) $[7,8]$. Likewise, several clinical studies have sought the role of fructose in the kidney, but the issue remains controversial. Interestingly, more than two SSB per day is associated with an incidence and a prevalence of chronic kidney disease (CKD) [9,10], but less than one SSB per day was not $[11,12]$. Fructose may therefore impair renal function in a dose-dependent manner. An intervention study also showed that a low-fructose diet lowered blood pressure and reduced systemic inflammation in subjects with CKD [13].

A recent scientific discovery is that fructose is produced endogenously, and is involved in the pathogenesis of several types of disorders. Acute kidney injury, diabetic nephropathy, cardiac hypertrophy, aging, and salt-sensitive hypertension are now recognized to be mediated by endogenous fructose.

This article summarizes the basis of fructose physiology, discusses a potential mechanism by which fructose causes kidney disease, and finally proposes our hypothesis that fructose mediates the Warburg effect in CKD.

\section{Current concepts and update regarding dietary fructose metabolism}

It has long been assumed that the liver is the primary site for dietary fructose metabolism, but recent studies have demonstrated that the small intestine plays a substantial role in dietary fructose metabolism [14]. After sucrose is digested by sucrase into fructose and glucose, fructose is absorbed by enterocytes via the glucose transporter (GLUT)
5 at the apical membrane of enterocytes. Since the intestine contributes $\sim 25 \%$ of systemic gluconeogenesis both after prolonged fasting and in diabetes [15], intestinal epithelium likely utilizes dietary fructose as a substrate for gluconeogenesis (Fig. 1). However, when present in excess, fructose saturates the intestinal metabolic capacity. Excessive fructose either spills over to the colon or is transported through the GLUT2 from the basal membrane into the portal vein and then to the liver [14]. Interestingly, intestinal fructose metabolism determines an individual's preference for sweet tastes and sugar intake but does not contribute to the development of metabolic syndrome [16]. In the colon, fructose is likely digested by microbiota that use fructose carbons to generate tricarboxylic acid (TCA) cycle intermediates, essential amino acids, and short-chain fatty acids [14]. In turn, fructose spilling over from intestinal shield acts on the hepatocyte via GLUT2 and drives the metabolic syndrome [16]. Hepatic fructose metabolism is associated with increased hepatic fatty acid and malonyl-CoA synthesis, reduced fatty acid oxidation, and modification of the mitochondrial proteome [17]. Similar to the enterocyte, excessive fructose in the kidney likely escapes into the systemic circulation. In the kidney, fructose in systemic circulation is filtered through the glomerulus into the urinary space, and urinary fructose is reabsorbed by the proximal tubular cells (Fig. 1).

\section{Fructose transporters are predominantly expressed in the proximal tubules}

After fructose is metabolized in the liver, only small amount of fructose escapes from the liver to reach the systemic circulation, and therefore serum fructose concentrations range from 0.1 to $0.8 \mathrm{mM}$ [18]. After filtration through the glomerulus, urinary fructose is either reabsorbed in the proximal tubular epithelial cells (Fig. 2), or excreted in the urine.

GLUT5, a high-affinity facilitative transporter, is considered to play a major role in fructose transport and is expressed at the apical membrane of the epithelial cells in the straight portion of the proximal tubule $[19,20]$. An alternate fructose transporter is the sodium glucose cotransporter 5 (SGLT5), which is a high-affinity transporter for fructose and mannose in humans and mice [21,22]. This transporter is exclusively expressed in the kidney, and likely located in the S2 segment of the proximal tubular cells [23]. The 


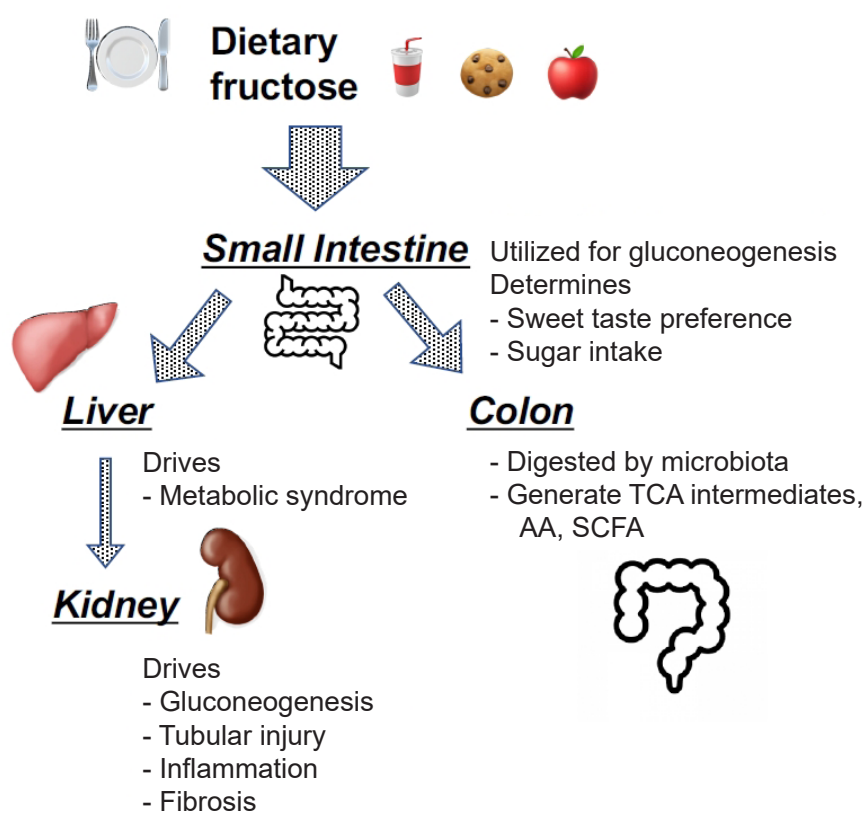

Figure 1. Current concepts regarding the metabolic pathways of dietary fructose. Dietary fructose is absorbed by enterocytes via glucose transporter (GLUT) 5 at the apical membrane of enterocytes and is likely utilized as a substrate for gluconeogenesis. Interestingly, intestinal fructose metabolism determines an individual's preference for sweet tastes and sugar intake but does not contribute to the development of the metabolic syndrome. When present in excess, fructose may saturate intestinal metabolic capacity and spill over to the colon where fructose can be digested by gut microbiota and utilized for the generation of tricarboxylic acid (TCA) intermediates, essential amino acids (AA), and short-chain fatty acids (SCFA). Alternatively, fructose exceeding the metabolic capacity of enterocytes is excreted from GLUT2 at the basal membrane and passes into the portal circulation, and reaches the hepatocytes. The fructose is then reabsorbed via GLUT2 at the surface of hepatocyte to be metabolized, likely driving the metabolic syndrome. Hepatic fructose metabolism is associated with increased hepatic fatty acid synthesis and malonyl-CoA levels, and a reduction in fatty acid oxidation. Similar to the enterocyte, excessive fructose likely escapes from hepatocyte into the systemic circulation. The kidney also plays a role in reabsorption and excretion of fructose. At physiological concentrations, fructose is utilized for gluconeogenesis, whereas it causes kidney injury when present in excess.

rat sodium-dependent glucose transporter 1 (rNaGLT1) is also expressed at the apical membrane of epithelial cells in both the convoluted and straight proximal tubules in the rat, and also mediates fructose transport [24]. While GLUT9 (SLC2A9) is a member of the facilitative GLUT gene family, it is now primarily described as a urate transporter (URAT) that can exchange both fructose and glucose for urate. The two splice variants of GLUT9, GLUT9a (full length) and GLUT9b $(\Delta \mathrm{N})$ are both present in the human kidney [25]. The GLUT9a (540 amino acids) splice variant is expressed in the basolateral membrane of the proximal tubular epithelial cells and favors urate transport back into the circulation from the tubular cells [26]. In turn, the GLUT9b (512 amino acids) splice variant is expressed at the apical site, and likely transfers urate from tubular fluid into cells $[26,27]$ and the collecting ducts $[25,28]$ in humans.

Alternatively, GLUT2 may transport fructose from the basolateral membrane of the proximal tubular cells into the systemic circulation [23,29], perhaps when fructose is abundant in the cytosol (Fig. 2). Given GLUT2 is a facilitative transporter operated by a passive diffusion process, it may act to excrete fructose when the intracellular fructose concentration is greater than that of the blood.

\section{Physiology of fructose metabolism in the proximal tubules}

The straight segment of the proximal tubules exclusively expresses GLUT5 so that it may be the primary site for fructose metabolism. However, both fructokinase and aldolase $\mathrm{B}$, another key enzyme for fructose metabolism, are also present in the convoluted proximal tubules [30,31], suggesting that fructose metabolism is not restricted in the straight segment, but is also likely operated in the convoluted segment of renal tubules.

Fructose are likely utilized as substrates for gluconeogenesis in the proximal tubules, where gluconeogenesis is dominant over glycolysis. In fact, several gluconeogenesis enzymes, including phosphoenolpyruvate carboxykinase, fructose bisphosphatase, and enzymes of the glucose 6-phosphatase system are dominantly activated [32,33], while glycolysis enzymes are less activated [34-36] in the proximal tubules compared to other parts of nephron.

In 1961, by utilizing in situ perfusion in the rat, Salomon et al. [37] directly measured the difference between arteriovenous fructose and glucose concentrations after bolus infusion of $25 \mathrm{mg}$ of fructose into the peripheral vessels. The reduction of fructose concentrations after passage of blood through the kidney was associated with equivalent increases in the renal venous glucose; the extent of fructose 


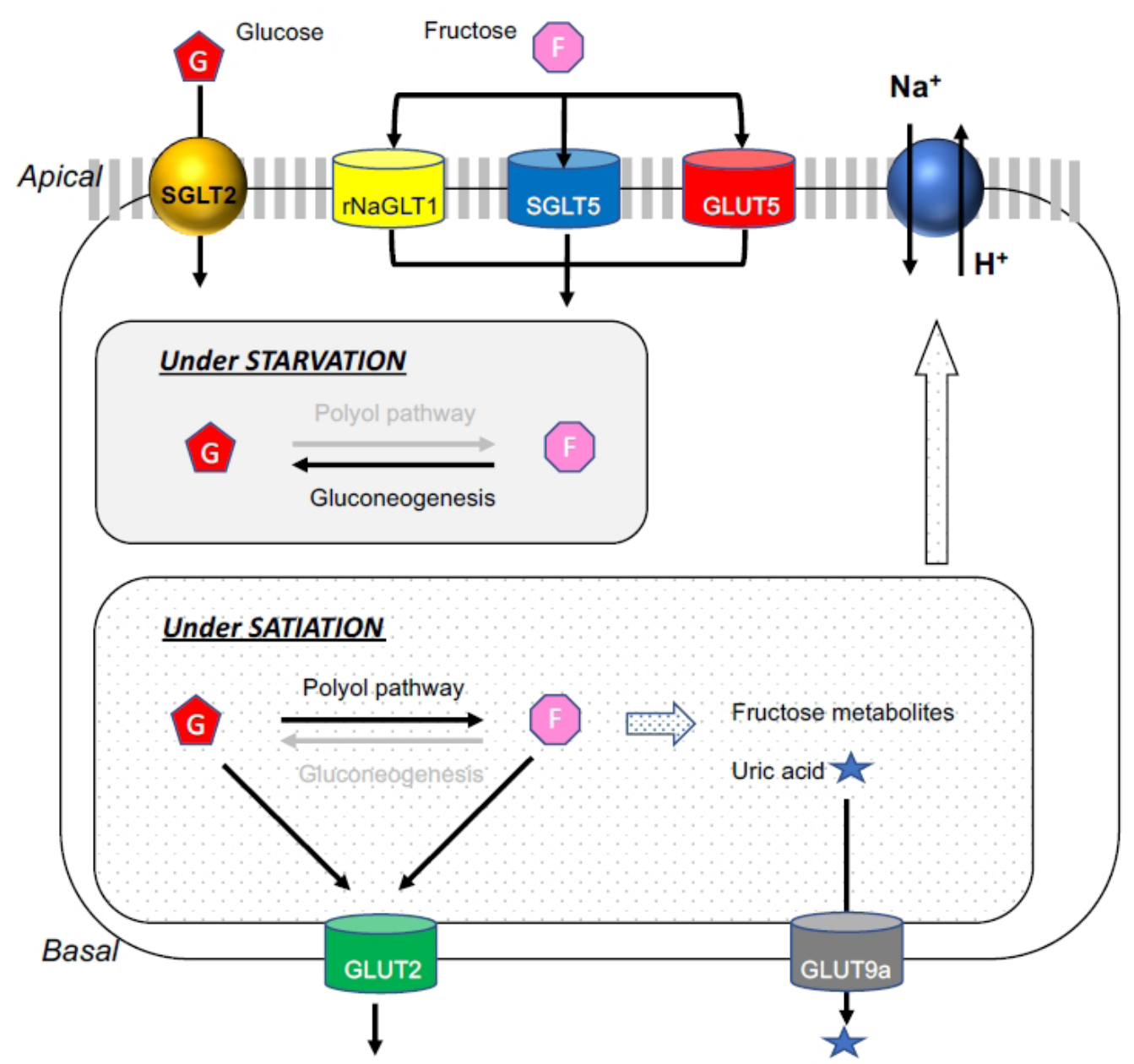

Figure 2. Fructose transporters and metabolism in the proximal tubules. In the proximal tubules, urinary fructose is reabsorbed at the apical membrane of the epithelial cells via several types of fructose transporters. The glucose transporter (GLUT) 5 is considered to be a major transporter for fructose. The sodium glucose cotransporter 5 (SGLT5) is a high-affinity kidney-specific transporter for fructose and mannose in humans and mice, while the rat sodium-dependent glucose transporter-1 (rNaGLT1) is also expressed in both convoluted and straight proximal tubules in the rat and also mediates fructose transport. Under physiological conditions or during starvation, fructose is utilized as a substrate for gluconeogenesis. In turn, during satiation or when fructose is in excess, fructose is metabolized in the cytosol to produce several fructose metabolites, including uric acid. The GLUT2 facilitative transporter is expressed in the basal membrane. When fructose concentrations are higher in the cytosol than in the blood of peritubular capillary, GLUT2 transports intracellular fructose into the blood in the peritubular capillary. Likewise, GLUT9a, another facilitative transporter, is expressed in the basolateral membrane and favors urate transport back into the circulation from the tubular cells.

disappearance and the appearance of glucose averaged approximately 19\%. In 1982, Björkman and Felig [38] found that intravenous infusion of fructose in humans at $2 \mathrm{mmol} /$ min for 135 minutes resulted in a rise in glucose concentration in the renal vein $(0.17 \pm 0.05 \mathrm{mmol} / \mathrm{L})$. The results indicated that $20 \%$ of intravenously infused fructose was taken up by the kidney, and the net glucose release from the kidney could be derived from $55 \%$ of the net renal uptake of fructose.

\section{Proximal tubular cells go wrong with excessive fructose}

When the proximal tubular cells are overloaded with excessive fructose after satiation, fructose metabolism likely becomes dysregulated and causes pathological reactions (Figs. 2, 3). In experimental studies, normal rats developed mild tubulointerstitial injury with inflammation and fibrosis when fed a high-fructose diet $[19,39]$. In the case of preex- 


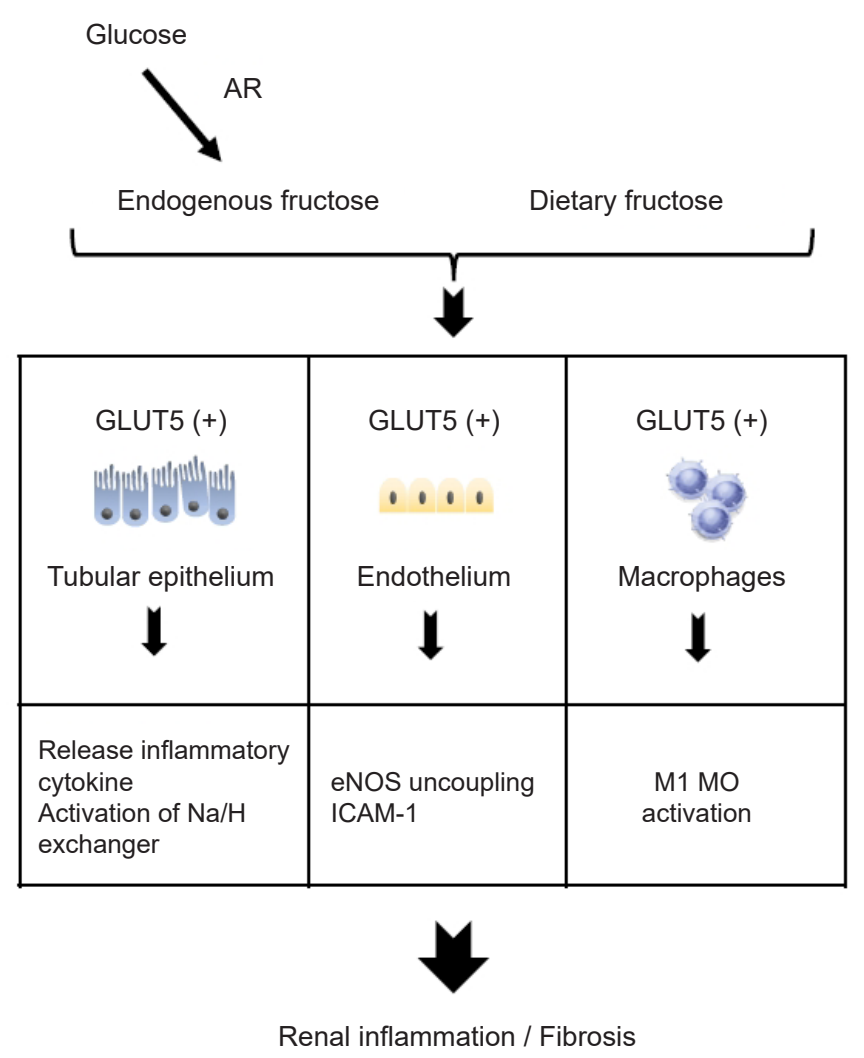

Figure 3. Postulate mechanism of fructose-induced kidney disease. Fructose, arising either from the diet or from endogenous production under pathological conditions, acts on the tubular epithelial cells, endothelial cells, and macrophages through fructose transporters, such as glucose transporter 5 (GLUT5), to cause inflammation and fibrosis in the kidney.

AR, aldose reductase; eNOS, endothelial NO synthase; ICAM-1, intercellular adhesion molecule-1; MO, macrophage.

isting kidney injury, fructose accelerates tubular injury and interstitial inflammation and fibrosis in CKD rats [40]. An in vitro study showed that the cultured proximal tubular cells released inflammatory cytokines, including monocyte chemoattractant protein-1, in response to pathological fructose concentrations; interestingly, this reaction was found to be mediated by uric acid [41].

Endothelial cells express GLUT5 [42] and release intercellular adhesion molecule-1 (ICAM-1) in response to fructose [43]. A likely mechanism for this effect is the ability of fructose to reduce nitric oxide (NO) availability due to uncoupling of endothelia nitric oxide synthase (eNOS), as NO donors mitigated the fructose-induced ICAM- 1 expression [43-45]. Fructose-induced generation of uric acid could be also involved in this process, as uric acid directly impairs endothelial function $[46,47]$.

Likewise, macrophages are also a target for fructose given they express GLUT5 (Fig. 3). Several experimental studies have shown that a high-fructose diet causes renal inflammation with macrophage infiltration in rodents $[19,40,43]$. In vitro studies confirmed the ability of fructose to act directly on macrophages via GLUT5 to release inflammatory cytokines $[48,49]$. Macrophages exhibit two phenotypes, a proinflammatory $\mathrm{M} 1$ and an anti-inflammatory M2 phenotype; fructose is likely an ideal fuel for M1 macrophage, which relies on glycolysis. However, the mechanism by which fructose activates macrophages may be somewhat complex. A recent study of fructose-induced inflammation demonstrated that oxidative metabolism, but not glycolysis, plays a dominant role in macrophage activation [48]. While a basic concept is that uric acid suppresses aconitase in the TCA cycle and hence reduces mitochondrial respiration, the investigators identified an alternative pathway for fructose to stimulate the TCA cycles. They found that glutamine was incorporated into the TCA cycles in response to fructose, and supplies $\alpha$-ketoglutarate that can bypass this step allowing oxidative phosphorylation to occur in human monocytes and mouse macrophages. Therefore, macrophages likely utilize either glycolysis or oxidative phosphorylation, perhaps depending on cellular conditions. While it remains unclear how macrophages switch these pathways, a key trigger may be oxygen availability. Under severe hypoxia, cytochrome c oxidase activity decreases [50], and glycolysis is dominant [51]. In contrast, under aerobic conditions, cytochrome c activity is activated, and glycolysis is completely replaced by oxidative phosphorylation [51].

\section{Fructose causes salt-sensitive hypertension}

Fructose intake is likely associated with hypertension in humans [52]. Experimental studies have shown that rats fed a high-fructose diet had elevated blood pressure in response to additional salt intake (Fig. 2). The proximal tubules play a key role in salt handling, as the majority of $\mathrm{Na}+$ filtered through glomerulus is reabsorbed into the tubular epithelial cells via $\mathrm{Na}+\mathrm{H}+$ exchangers (NHEs) located in the apical membrane [53,54]. Fructose-induced salt sensitivity can be accounted for by the ability of fructose to stimulate both 
the expression and the activity of NHEs, and increase $\mathrm{Na}+$ reabsorption in the proximal tubules [53]. While NHEs are regulated by angiotensin II, fructose sensitizes the proximal tubules to angiotensin II by upregulating NHE expression $[53,54]$. In addition, urate also plays a key role in the development of salt-sensitive hypertension in response to fructose as it causes arteriolopathy, tubulointerstitial injury, and a reduction in NO in endothelial cells $[43,46,47,55]$.

\section{Renal proximal tubular cells turn on glycolysis when injured}

The proximal tubular cells normally prefer lipids over glucose for energy generation, so glycolysis is not operative in this cell type. It is because enzymes for gluconeogenesis are dominantly activated over glycolytic enzymes; and therefore, fructose metabolism is physiologically linked with gluconeogenesis, but not with glycolysis [56]. However, this is unlikely the case when the tubular cells are damaged. In fact, damaged proximal tubular cells are often associated with mitochondrial alteration, resulting in a metabolic switch from mitochondrial oxidative phosphorylation to glycolysis with amplified expression of glycolytic enzymes [57]. Thus, both fructose and glucose are metabolized in damaged proximal tubular cells.

\section{Does the combination of fructose with glucose accelerate glycolysis in renal proximal tubular epithelial cells?}

The combination of fructose with glucose modifies the activation of glucokinase, the enzyme that catalyzes the first step of glycolysis. In hepatocytes, glucokinase is positively regulated by fructose 1-phosphate (FrulP) whereas it is inhibited by fructose 6-phosphate (Fru6P) [58,59]. The mechanism for FrulP-mediated glucokinase activation is the release of glucokinase from glucokinase regulatory protein (GKRP), which sequesters glucokinase in the nucleus [60,61]. Even at low concentrations, intracellular fructose is rapidly metabolized to FrulP. Therefore, FrulP-induced glucokinase activation may explain how fructose facilitates glucose utilization. Consistent with these findings, Shiota et al. [62] showed that small amounts of fructose enhanced hepatic glucose uptake in the dog. Furthermore, fructose metabolism also increases fructokinase activity, which de- pletes intracellular adenosine triphosphate (ATP). Since ATP negatively regulates the glycolytic pathway by inhibiting phosphofructokinase and pyruvate kinase, the ATP depletion due to fructokinase activation enhances glycolysis. However, these processes may not occur in the kidney, given glucokinase (hexokinase IV) is expressed only in hepatocytes and pancreatic $\beta$ cells, [63] while renal proximal tubular cells express hexokinase I and II $[57,64]$.

\section{Endogenous fructose may be a unifying pathway in the development of chronic kidney diseases}

Interestingly, fructose is produced endogenously in the kidney, particularly under condition of ischemia/hypoxia, high osmotic stress, aging, pressure overload, and diabetes. A potential mechanism for fructose synthesis is the activation of the polyol pathway, in which glucose is reduced by aldose reductase to sorbitol, which is then oxidized by sorbitol dehydrogenase to fructose. Therefore, fructose can be readily produced when glucose is constantly supplied. A key step in this process is the activation of aldose reductase, which can be stimulated by several factors, including hypoxia, osmotic stress, and diabetes, and may also account for the findings that several factors induce endogenous fructose production in several pathological conditions.

Cardiac surgery often results in postoperative acute kidney injury due to ischemia. Our research group studied pediatric patients who underwent cardiac bypass surgery and found that urinary fructose concentrations were elevated in patients with ischemic acute kidney injury (iAKI) compared with patients without iAKI [65]. Mice with iAKI also exhibited increased renal fructose concentrations [65], suggesting that ischemia could stimulate endogenous production of fructose in the kidney. Similarly, compared with nondiabetic mice, diabetic mice also had higher fructose levels in the kidney due to the activation of the polyol pathway [66]. Importantly, both studies demonstrated that blocking fructose metabolism ameliorated tubular injury induced by either ischemia or diabetes in mice lacking the fructokinase gene $[65,66]$.

In the senescent kidney, endogenous fructose production likely contributes to the development of glomerular injury. A mouse study showed that glomerular injury accompanied by glomerular hypertrophy, collagen IV deposition, and mesangiolysis was observed in aging wild-type 
mice while aging fructokinase-knockout mice developed significantly less glomerular injury [67]. In turn, high salt intake also stimulates endogenous fructose production in the liver, while blocking fructokinase slows fat accumulation in the epididymis [68]. In addition, in the mouse heart, pressure-overload-induced cardiac hypertrophy was ameliorated by blocking fructose metabolism [69].

\section{Pathways downstream from fructose metabolism may contribute to kidney disease}

In the kidney, inflammation and fibrosis are accompanied with several pathological steps, including cell proliferation, enzymatic activation, and protein synthesis, which require several biological factors, including energy sources, nucleotides, lipids, and redox balance, which can be efficiently provided by aberrant glycolysis. Fructose is metabolized through several pathways and contributes to the progression of CKD (Fig. 4).

\section{Glycolysis}

The first enzyme involved in fructose metabolism is fructokinase (known as ketohexokinase [KHK]), which phosphorylates fructose to produce FrulP (Fig. 4). There are two spliced isoforms of KHK, and each is produced by mutual exclusion of the adjacent exons $3 \mathrm{C}$ and $3 \mathrm{~A}$ within the $K H K$ gene [70]. The " $\mathrm{A}$ " isoform is ubiquitously expressed but has low activity due to relatively low affinity for its substrate (Km $8 \mathrm{mM}$ ) [71]. Expression of the "C" isoform is primarily restricted to metabolic tissues, including the liver, kidney, and intestine, and this form has much higher affinity for fructose $(\mathrm{Km} 0.8 \mathrm{mM})[71,72]$. FrulP is subsequently metabolized by aldolase B and triokinase to dihydroxyacetone phosphate and glyceraldehyde-3-phosphate that enter the glycolytic pathway downstream of phosphofructokinase. Subsequently, glyceraldehyde-3-phosphate is metabolized to pyruvate in the glycolytic pathway to produce ATP and nicotinamide adenine dinucleotide. Pyruvate is further converted into lactate by lactate dehydrogenase. Importantly, this reaction is usually stimulated by low oxygen, but is accelerated by fructose even under aerobic condition [73]. Lactate seems to be an energy for cancer growth [74].

\section{Pentose phosphate pathway}

The pentose phosphate pathway (PPP) is activated by fructose and comprises two distinct phases, the oxidative pathway and the non-oxidative pathway (Fig. 4). Glucose 6-phosphate, a fructose metabolite, is metabolized by three sequential reactions in the oxidative pathway to NAPDH, which supplies reducing equivalents, and reduces glutathione through the action of glutathione reductase. In turn, two forms of fructose carbon backbones, Fru6P and glyceraldehyde-3-phosphate, are catalyzed by transketolase to enter the non-oxidative pathway for nucleotide formation through ribose 5-phosphate, while erythrose 4-phosphate is metabolized into amino acids. Alternatively, activated hexokinase can convert fructose into Fru6P, which may be a link between glycolysis and the nonoxidative PPP in cancer cells [75].

\section{Lipogenesis}

Lipids are required as an energy source, for membrane formation, and as signaling molecules (Fig. 4). Fructose is metabolized in the glycolytic pathway to provide acetyl-CoA as the building block of carbon chains for de novo lipogenesis, and also promotes fatty acid synthesis to form palmitate. In turn, glyceraldehyde-3-phosphate, carrying a fructose-based carbon backbone, is also utilized to form triglycerides. Fructose also stimulates intracellular signaling pathways, including those mediated by carbohydrate-responsive element-binding protein [76] and GKRP [60]. A recent study using a mouse model demonstrated that fructose-mediated fatty liver disease was likely mediated by impairment of fatty acid oxidation due to an increased acetylation of long-chain specific acyl-CoA dehydrogenase and carnitine palmitoyl-transferase $1 \alpha$ [17].

\section{Uric acid production and the Warburg effect}

Fructokinase activation rapidly sequesters phosphate, consequently activating adenosine monophosphate (AMP) deaminase to cleave AMP to inosine monophosphate (IMP) (Fig. 4). However, phosphate levels subsequently increase due to the slower reaction of aldolase with FrulP. This reaction is further accentuated by the increased IMP, which is an aldolase B inhibitor [77]. Sequential enzymatic acti- 


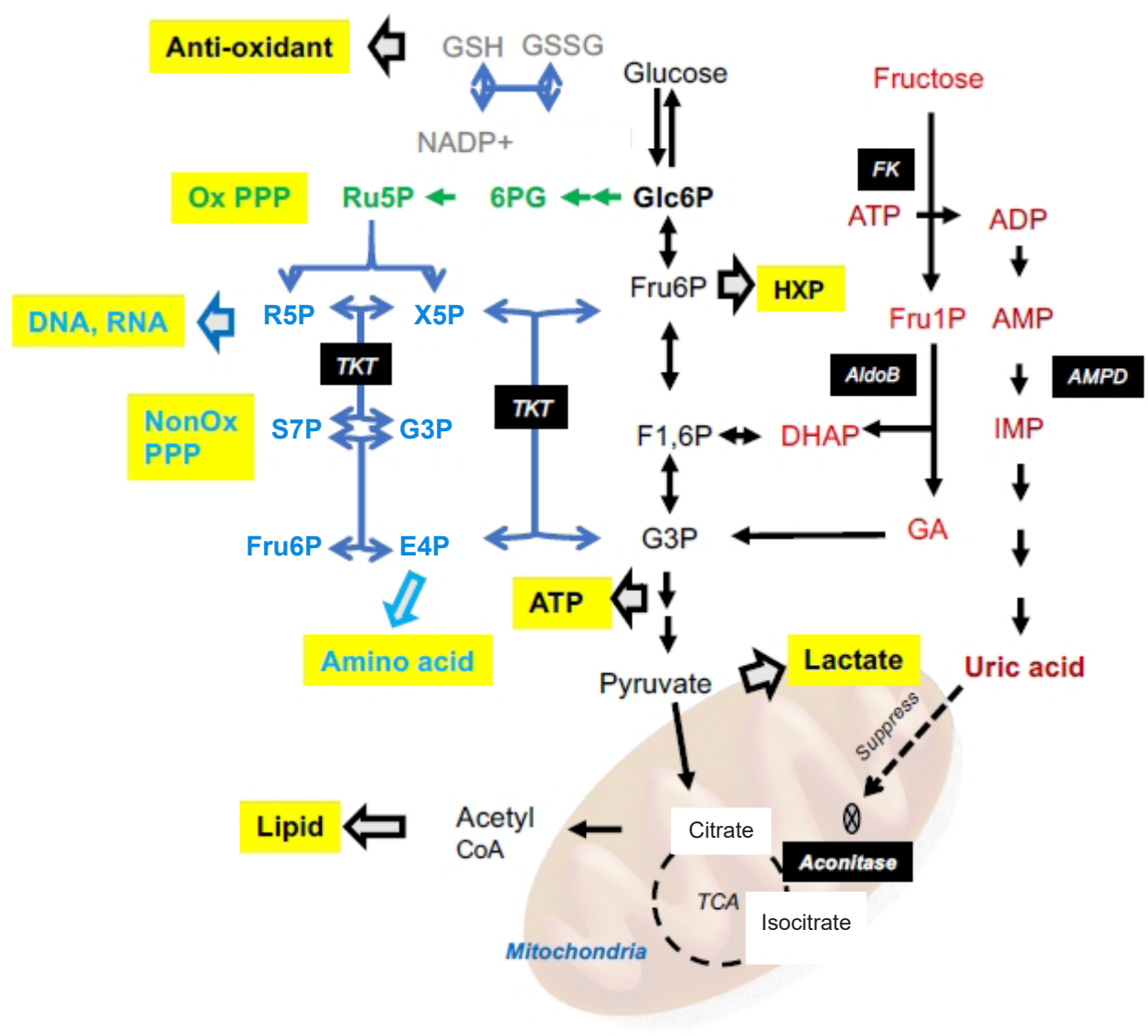

Figure 4. Several pathways downstream from fructose metabolism. Fructose is initially metabolized to fructose 1-phosphate by fructokinase, which rapidly sequesters phosphate, consequently activating adenosine monophosphate (AMP) deaminase to cleave AMP to inosine monophosphate (IMP). Sequential enzymatic activation metabolizes IMP and eventually produces uric acid. Uric acid subsequently inhibits aconitase in the tricarboxylic acid (TCA) cycle to suppress mitochondrial respiration. Glycolysis is preferentially activated, and several metabolites are fed into several side pathways, including the pentose phosphate pathway (PPP) and hexosamine pathway (HXP), which aberrantly activate energy production, synthesis of biosynthetic precursors, and redox homeostasis.

ADP, adenosine diphosphate; AldoB, aldorase B; ATP, adenosine triphosphate; DHAP, dihydroxyacetone phosphate; E4P, erythrose 4-phosphate; F1,6P, fructose 1,6-biphosphate; FK, fructokinase; Fru1P, fructose 1-phosphate; Fru6P, fructose 6-phosphate; G3P, glyceraldehyde 3-phosphate; GA, glyceraraldehyde; Glc6P, glucose 6-phosphate; GSH, glutathione; GSSH, glutathione-S-S-glutathione; $\mathrm{NADP}+$, nicotinamide adenine dinucleotide phosphate; NonOx, non-oxidative; Ox, oxidative; R5P, ribose 5-phosphate; Ru5P, ribulose 5-phosphate; S7P, sedoheptulose 7-phosphate; TKT, transketolase; X5P, xylulose 5-phosphate; 6PG, 6-phosphogluconate.

vation metabolizes IMP and eventually produces uric acid. We found that uric acid could prevent the entry of fructose metabolites into mitochondrial oxidation in the human hepatocellular carcinoma cell line HepG2 [78]. A potential mechanism for this effect is the suppression of mitochondrial aconitase activity by uric acid, and disconnection of fructose metabolites from mitochondrial oxidation. Since aconitase lies at the junction of acetyl-CoA oxidation, blocking aconitase leads to acetyl-CoA shuttling out of the mitochondria, resulting in the accumulation of citrate in the cytosol. Citrate is then utilized for lipid synthesis by sequential ATP-citrate lyase and fatty-acid synthase [78]. As a result, fructose leads to a state of metabolic imbalance that favors glycolysis over mitochondrial respiration, resembling the Warburg effect in cancer [79].

\section{The Warburg effect is shared by non-cancer disorders}

In 1924, Otto Warburg initially described that cancer cells, 
as opposed to normal cells, exhibit a unique ability to ferment glucose to lactate even in the presence of sufficient oxygen [80]. This process is now recognized as a key mechanism of cancer growth and is called the "Warburg effect." However, we need to be cautious for when interpreting this effect, as general scientists tend to share a misconception regarding oxidative metabolism in the mitochondria [81]. Warburg's own experiments revealed persistent oxygen consumption in tumor tissues. The rate of mitochondrial respiration was low relative to what might have been predicted given the high rate of glucose uptake, but respiration itself did not appear to be impaired [81]. The Warburg effect was long considered a unique characteristic of cancer; however, recent studies indicate that aberrant glycolysis is not specific to cancer, but rather is shared by other non-cancer disorders [82].

The Warburg effect is involved in multiple processes in several disorders, and the cardiovascular, immune, and neuronal systems are now found to be all modulated by aerobic glycolysis [82]. Although glycolysis produces less ATP than mitochondrial oxidative phosphorylation, the process of aerobic glycolysis is much faster than that of mitochondrial respiration [83]. As a result, aerobic glycolysis can produce more ATP than mitochondrial oxidative phosphorylation in the same amount of time [84]. More importantly, the Warburg effect may impact more than energy production, and may regulate several cellular functions, including cell proliferation, extracellular matrix production, autophagy, and apoptosis [85], and may consequently participate in multiple biological processes.

\section{The Warburg effect is involved in kidney diseases}

Recent studies have documented that autosomal-dominant polycystic kidney disease (ADPKD) is mediated by aberrant glycolysis. Rowe et al. [86] demonstrated that cultured mouse embryonic fibroblasts derived from $P \mathrm{Pd}^{-/}$mice exhibited activated glycolysis, given the cells preferentially utilized greater amounts of glucose and excreted more lactate into the culture medium than cells from wild-type mice. Mice lacking $P k d$ in the renal tubules, as a mouse model of ADPKD, exhibited glycolysis activation while blocking glycolysis with 2-deoxy-D-glucose (2DG), a glucose analog, attenuated tubular cell proliferation, leading to the reduction in kidney size and cyst formation [87].
In diabetic nephropathy, mitochondrial overproduction of superoxide due to the activation of the electron transport chain is considered a unifying mechanism, but this hypothesis remains controversial [88]. Recent studies have demonstrated that mitochondrial function is suppressed in diabetic nephropathy, and the restoration of normal mitochondrial health improves renal, cardiovascular, and neuronal outcomes. In addition, mitochondrial TCA cycle metabolites are also significantly reduced in patients with diabetic nephropathy compared to healthy controls [89]. In turn, glycolytic activation is upregulated in the renal cortex in type 2 diabetes [90], suggesting that activation of glycolysis is dominant over mitochondrial oxidation and plays a pathological role in diabetic nephropathy.

A shift to glycolysis has also been observed in two animal models; one is a model of unilateral ureteral obstruction and the other is a transforming growth factor (TGF)- $\beta 1$-induced renal fibrosis model. Specifically, Ding et al. [91] found that myofibroblast activation in the kidneys was associated with enhanced renal glucose uptake and lactate production that could be attenuated by blocking glycolysis by 2DG treatment. In these models, a key factor is likely TGF- $\beta 1$ as this growth factor was capable of switching metabolic profile favoring glycolysis over mitochondrial respiration in fibroblasts. In addition to TGF- $\beta 1$, PDGF also causes the Warburg effect [92], consistent with the notion that growth factors disproportionately activate glycolysis relative to mitochondrial oxidation [93].

\section{Could natural fruit exacerbate kidney disease?}

One might ask whether fruit can be also deleterious to the kidney, given fruit contains substantial amount of fructose. This question arises from the assumption that fructose in natural fruit is theoretically metabolized, resulting in uric acid production, and therefore a large amount of fruit may be deleterious. In this regard, several clinical studies have examined the effect of fruit on renal function, and in many cases, the effect of consuming fruits together with vegetables, low salt, and other dietary modifications were assessed $[94,95]$. These studies generally found that our assumption was flawed and fruit was protective due to the improvement of metabolic acidosis, reduction of blood pressure, and prevention of cardiovascular diseases. However, the effect of a defined amount of fruits was examined in those studies, 
and it remains uncertain if a large amount of fruits could cause renal disease.

The mechanism by which fruits are protective of the kidney may be that metabolism of fructose in fruit is inhibited by vitamin $\mathrm{C}$ and other nutrients. For example, fructose-associated uric acid production is linked with xanthine oxidase activation and oxidant stress, which can be blocked by flavonoids/catechins and vitamin C in fruits [96]. Vitamin C also enhances urinary urate excretion through URAT-1 $[97,98]$ and lessens the effects of uric acid. In addition, the potassium present in many fruits can ameliorate urate-induced endothelial dysfunction [99].

In this regard, we previously discussed this issue and reviewed the effect of variety of natural fruits on hyperuricemia or gout [100]. One thing to bear in mind is that the effects of fruit are often inconsistent in clinical studies, in part due to difference in study designs, although other factors may impact the results. Fruit intake is often estimated from the results of face-to-face interviews or questionnaires, which usually rely on memory, and may not always be accurate [101]. The composition of fruits may vary significantly depending on growing, harvesting, and storage conditions, and how they are prepared for consumption. For example, black currants become sweeter at higher growing temperatures and their taste varies with season, with the concentrations of fructose, glucose, and vitamin C found
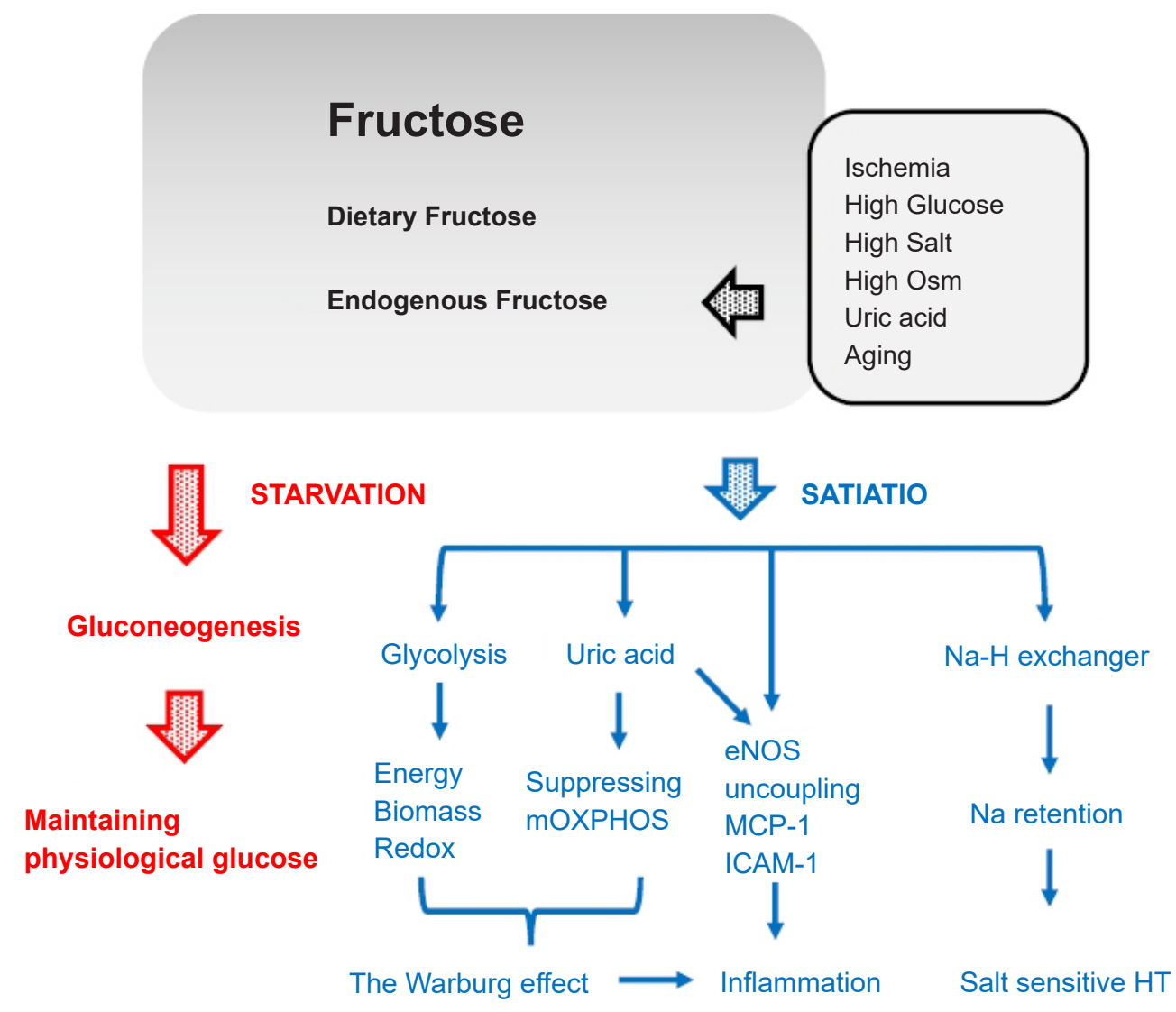

Figure 5. Current hypothesis of the pathophysiology of fructose in the kidney. In addition to dietary fructose, fructose can be produced endogenously as a result of several pathological conditions in the kidney. During starvation, fructose is utilized for gluconeogenesis in the proximal tubular cells and excreted into the systemic circulation to maintain serum glucose concentrations. In turn, when in excess or during satiation, fructose is associated with aberrant energy production, biomass synthesis, and redox balance with uric acid production, resulting in the Warburg effect in the kidney. Together with such reactions, fructose also causes endothelial NO synthase (eNOS) uncoupling in endothelial cells and release inflammatory cytokines. Fructose also stimulates the $\mathrm{Na} / \mathrm{H}$ exchanger to accelerate sodium absorption, leading to salt-sensitive hypertension (HT).

ICAM-1, intercellular adhesion molecule-1; MCP-1, monocyte chemoattractant protein-1, mOXPHOS, mitochondrial oxidative phosphorylation; Osm, osmolarity. 
to also differ depending upon the season [102]. In addition, humidity and latitude influence the maturation process of many fruits, and often determine the sugar content; together, these various influences may result in sweeter fruit in the fall than in the spring, and greater fructose content in mature fruit than less mature fruit [103]. These factors may add a layer of complexity to the effects of fruit intake on human renal disease.

\section{Conclusions}

Several risk factors, including hypoxia, high blood glucose concentrations, senescence, and cardiac pressure overload, are found to share endogenous fructose production in the kidney as a common underlying factor (Fig. 5). A unique characteristic of fructose metabolism, as opposed to glucose metabolism, is the production of uric acid. Excessive uric acid production links to inflammation, endothelial dysfunction, vascular injury, and insulin resistance, while also favoring glycolysis over mitochondrial respiration, similar to the Warburg effect in cancer. The Warburg effect creates a pool of biosynthetic precursors that contribute to several pathological processes, including nucleotide synthesis, amino acid production, lipids and lactate, and excessive endogenous fructose is likewise a mechanism of CKD (Fig. 5). Further studies exploring the role of endogenous fructose in CKD are warranted.

\section{Conflicts of interest}

Takahiko Nakagawa has equity with XORTX therapeutics, which is developing novel xanthine oxidase inhibitors. The authors have no other conflicts of interest to declare.

\section{Funding}

This work was supported by a grant from the Korean Society of Nephrology (BAXTER, 2017) and a National Research Foundation of Korea (NRF) grant funded by the Korean government MIST, (2020R1A2C3007759).

\section{Authors' contributions}

Conceptualization: $\mathrm{TN}$

Writing-original draft: TN
Writing-review \& editing: DHK

All authors read and approved the final manuscript.

\section{ORCID}

Takahiko Nakagawa, https://orcid.org/0000-0002-0127-1529

Duk-Hee Kang, https://orcid.org/0000-0001-8475-8932

\section{References}

1. Johnson RJ, Andrews P, Benner SA, Oliver W. Theodore E. Woodward award: the evolution of obesity: insights from the mid-Miocene. Trans Am Clin Climatol Assoc 2010;121:295-308.

2. Johnson RJ, Stenvinkel P, Andrews P, et al. Fructose metabolism as a common evolutionary pathway of survival associated with climate change, food shortage and droughts. J Intern Med 2020;287:252-262.

3. Park TJ, Reznick J, Peterson BL, et al. Fructose-driven glycolysis supports anoxia resistance in the naked mole-rat. Science 2017;356:307-311.

4. Bacon JS, Bell DJ. The identification of fructose as a constituent of the foetal blood of the sheep. Biochem J 1946;40:xlii.

5. Goodwin RF. Division of the common mammals into two groups according to the concentration of fructose in the blood of the foetus. J Physiol 1956;132:146-156.

6. Jauniaux E, Hempstock J, Teng C, Battaglia FC, Burton GJ. Polyol concentrations in the fluid compartments of the human conceptus during the first trimester of pregnancy: maintenance of redox potential in a low oxygen environment. J Clin Endocrinol Metab 2005;90:1171-1175.

7. Johnson RJ, Segal MS, Sautin Y, et al. Potential role of sugar (fructose) in the epidemic of hypertension, obesity and the metabolic syndrome, diabetes, kidney disease, and cardiovascular disease. Am J Clin Nutr 2007;86:899-906.

8. Neilson EG. The fructose nation. J Am Soc Nephrol 2007;18: 2619-2621.

9. Saldana TM, Basso O, Darden R, Sandler DP. Carbonated beverages and chronic kidney disease. Epidemiology 2007;18:501506.

10. Rebholz CM, Young BA, Katz R, et al. Patterns of beverages consumed and risk of incident kidney disease. Clin J Am Soc Nephrol 2019;14:49-56.

11. Bomback AS, Katz R, He K, Shoham DA, Burke GL, Klemmer PJ. Sugar-sweetened beverage consumption and the progression of chronic kidney disease in the Multi-Ethnic Study of Atheroscle- 
rosis (MESA). Am J Clin Nutr 2009;90:1172-1178.

12. Sundborn G, Thornley S, Merriman TR, et al. Are liquid sugars different from solid sugar in their ability to cause metabolic syndrome? Obesity (Silver Spring) 2019;27:879-887.

13. Brymora A, Flisiński M, Johnson RJ, Goszka G, Stefańska A, Manitius J. Low-fructose diet lowers blood pressure and inflammation in patients with chronic kidney disease. Nephrol Dial Transplant 2012;27:608-612.

14. Jang C, Hui S, Lu W, Cowan AJ, Morscher RJ, Lee G, et al. The small intestine converts dietary fructose into glucose and organic acids. Cell Metab 2018;27:351-361.

15. Soty M, Penhoat A, Amigo-Correig M, Vinera J, Sardella A, Vullin-Bouilloux F, et al. A gut-brain neural circuit controlled by intestinal gluconeogenesis is crucial in metabolic health. Mol Metab 2014;4:106-117.

16. Andres-Hernando A, Orlicky DJ, Kuwabara M, et al. Deletion of fructokinase in the liver or in the intestine reveals differential effects on sugar-induced metabolic dysfunction. Cell Metab 2020;32:117-127.

17. Softic S, Meyer JG, Wang GX, et al. Dietary sugars alter hepatic fatty acid oxidation via transcriptional and post-translational modifications of mitochondrial proteins. Cell Metab 2019; 30:735-753.

18. Macdonald I, Keyser A, Pacy D. Some effects, in man, of varying the load of glucose, sucrose, fructose, or sorbitol on various metabolites in blood. Am J Clin Nutr 1978;31:1305-1311.

19. Nakayama T, Kosugi T, Gersch M, et al. Dietary fructose causes tubulointerstitial injury in the normal rat kidney. Am J Physiol Renal Physiol 2010;298:F712-F720.

20. Chin E, Zhou J, Bondy C. Anatomical and developmental patterns of facilitative glucose transporter gene expression in the rat kidney. J Clin Invest 1993;91:1810-1815.

21. Grempler R, Augustin R, Froehner S, et al. Functional characterisation of human SGLT-5 as a novel kidney-specific sodium-dependent sugar transporter. FEBS Lett 2012;586:248-253.

22. Fukuzawa T, Fukazawa M, Ueda O, et al. SGLT5 reabsorbs fructose in the kidney but its deficiency paradoxically exacerbates hepatic steatosis induced by fructose. PLoS One 2013;8:e56681.

23. Gonzalez-Vicente A, Cabral PD, Hong NJ, Asirwatham J, Saez F, Garvin JL. Fructose reabsorption by rat proximal tubules: role of $\mathrm{Na}+$-linked cotransporters and the effect of dietary fructose. Am J Physiol Renal Physiol 2019;316:F473-F480.

24. Horiba N, Masuda S, Ohnishi C, Takeuchi D, Okuda M, Inui K. $\mathrm{Na}(+)$-dependent fructose transport via rNaGLT1 in rat kidney. FEBS Lett 2003;546:276-280.
25. Augustin R, Carayannopoulos MO, Dowd LO, Phay JE, Moley JF, Moley KH. Identification and characterization of human glucose transporter-like protein-9 (GLUT9): alternative splicing alters trafficking. J Biol Chem 2004;279:16229-16236.

26. Matsuo H, Chiba T, Nagamori S, et al. Mutations in glucose transporter 9 gene SLC2A9 cause renal hypouricemia. Am J Hum Genet 2008;83:744-751.

27. So A, Thorens B. Uric acid transport and disease. J Clin Invest 2010;120:1791-1799.

28. Kimura T, Takahashi M, Yan K, Sakurai H. Expression of SLC2A9 isoforms in the kidney and their localization in polarized epithelial cells. PLoS One 2014;9:e84996.

29. Marks J, Carvou NJ, Debnam ES, Srai SK, Unwin RJ. Diabetes increases facilitative glucose uptake and GLUT2 expression at the rat proximal tubule brush border membrane. J Physiol 2003;553(Pt 1):137-145.

30. Burch HB, Choi S, Dence CN, Alvey TR, Cole BR, Lowry OH. Metabolic effects of large fructose loads in different parts of the rat nephron. J Biol Chem 1980;255:8239-8244.

31. Kranhold JF, Loh D, Morris RC Jr. Renal fructose-metabolizing enzymes: significance in hereditary fructose intolerance. Science 1969;165:402-403.

32. Coffee EM, Tolan DR. Gluconeogenesis. In: Lee B, Scaglia F, editors. Inborn errors in metabolism: from neonatal screening to metabolic pathways. New York: Oxford University Press; 2014. p. 68-91.

33. Alleyne GA, Scullard GH. Renal metabolic response to acid base changes. I. Enzymatic control of ammoniagenesis in the rat. $J$ Clin Invest 1969;48:364-370.

34. Guder WG, Ross BD. Enzyme distribution along the nephron. Kidney Int 1984;26:101-111.

35. Schmidt U, Guder WG. Sites of enzyme activity along the nephron. Kidney Int 1976;9:233-242.

36. Schmidt U, Marosvari I, Dubach UC. Renal metabolism of glucose: anatomical sites of hexokinase activity in the rat nephron. FEBS Lett 1975;53:26-28.

37. Salomon LL, Lanza FL, Smith DE. Renal conversion of fructose to glucose. Am J Physiol 1961;200:871-877.

38. Björkman O, Felig P. Role of the kidney in the metabolism of fructose in 60-hour fasted humans. Diabetes 1982;31(6 Pt 1):516-520.

39. Aoyama M, Isshiki K, Kume S, et al. Fructose induces tubulointerstitial injury in the kidney of mice. Biochem Biophys Res Commun 2012;419:244-249.

40. Gersch MS, Mu W, Cirillo P, et al. Fructose, but not dextrose, accelerates the progression of chronic kidney disease. Am J Physiol 
Renal Physiol 2007;293:F1256-F1261.

41. Cirillo P, Gersch MS, Mu W, et al. Ketohexokinase-dependent metabolism of fructose induces proinflammatory mediators in proximal tubular cells. J Am Soc Nephrol 2009;20:545-553.

42. Giansante C, Fiotti N. Insights into human hypertension: the role of endothelial dysfunction. J Hum Hypertens 2006;20:725-726.

43. Glushakova O, Kosugi T, Roncal C, et al. Fructose induces the inflammatory molecule ICAM-1 in endothelial cells. J Am Soc Nephrol 2008;19:1712-1720.

44. Nakagawa T. Uncoupling of the VEGF-endothelial nitric oxide axis in diabetic nephropathy: an explanation for the paradoxical effects of VEGF in renal disease. Am J Physiol Renal Physiol 2007;292:F1665-F1672.

45. Nakagawa T, Tanabe K, Croker BP, et al. Endothelial dysfunction as a potential contributor in diabetic nephropathy. Nat Rev Nephrol 2011;7:36-44.

46. Khosla UM, Zharikov S, Finch JL, et al. Hyperuricemia induces endothelial dysfunction. Kidney Int 2005;67:1739-1742.

47. Nakagawa T, Hu H, Zharikov S, et al. A causal role for uric acid in fructose-induced metabolic syndrome. Am J Physiol Renal Physiol 2006;290:F625-F631.

48. Jones N, Blagih J, Zani F, et al. Fructose reprogrammes glutamine-dependent oxidative metabolism to support LPS-induced inflammation. Nat Commun 2021;12:1209.

49. Choe JY, Kim SK. Quercetin and ascorbic acid suppress fructose-induced NLRP3 inflammasome activation by blocking intracellular shuttling of TXNIP in human macrophage cell lines. Inflammation 2017;40:980-994.

50. Wilson DF, Rumsey WL, Green TJ, Vanderkooi JM. The oxygen dependence of mitochondrial oxidative phosphorylation measured by a new optical method for measuring oxygen concentration. J Biol Chem 1988;263:2712-2718.

51. Semba H, Takeda N, Isagawa T, et al. HIF-1a-PDK1 axis-induced active glycolysis plays an essential role in macrophage migratory capacity. Nat Commun 2016;7:11635.

52. Nguyen S, Choi HK, Lustig RH, Hsu CY. Sugar-sweetened beverages, serum uric acid, and blood pressure in adolescents. J Pediatr 2009;154:807-813.

53. Cabral PD, Hong NJ, Hye Khan MA, et al. Fructose stimulates $\mathrm{Na} / \mathrm{H}$ exchange activity and sensitizes the proximal tubule to angiotensin II. Hypertension 2014;63:e68-e73.

54. Gonzalez-Vicente A, Cabral PD, Hong NJ, et al. Dietary fructose enhances the ability of low concentrations of angiotensin II to stimulate proximal tubule $\mathrm{Na}+$ reabsorption. Nutrients 2017;9:885.
55. Watanabe S, Kang DH, Feng L, et al. Uric acid, hominoid evolution, and the pathogenesis of salt-sensitivity. Hypertension 2002;40:355-360.

56. Nakagawa T, Johnson RJ, Andres-Hernando A, et al. Fructose production and metabolism in the kidney. J Am Soc Nephrol 2020;31:898-906.

57. Lan R, Geng H, Singha PK, et al. Mitochondrial pathology and glycolytic shift during proximal tubule atrophy after ischemic AKI. J Am Soc Nephrol 2016;27:3356-3367.

58. Van Schaftingen E, Detheux M, Veiga da Cunha M. Short-term control of glucokinase activity: role of a regulatory protein. FASEB J 1994;8:414-419.

59. Agius L, Peak M. Intracellular binding of glucokinase in hepatocytes and translocation by glucose, fructose and insulin. Biochem J 1993;296(Pt 3):785-796.

60. Brown KS, Kalinowski SS, Megill JR, Durham SK, Mookhtiar KA. Glucokinase regulatory protein may interact with glucokinase in the hepatocyte nucleus. Diabetes 1997;46:179-186.

61. Niculescu L, Veiga-da-Cunha M, Van Schaftingen E. Investigation on the mechanism by which fructose, hexitols and other compounds regulate the translocation of glucokinase in rat hepatocytes. Biochem J 1997;321(Pt 1):239-246.

62. Shiota M, Galassetti P, Monohan M, Neal DW, Cherrington AD. Small amounts of fructose markedly augment net hepatic glucose uptake in the conscious dog. Diabetes 1998;47:867-873.

63. Mathupala SP, Ko YH, Pedersen PL. Hexokinase II: cancer's double-edged sword acting as both facilitator and gatekeeper of malignancy when bound to mitochondria. Oncogene 2006;25:47774786.

64. Gall JM, Wong V, Pimental DR, et al. Hexokinase regulates Bax-mediated mitochondrial membrane injury following ischemic stress. Kidney Int 2011;79:1207-1216.

65. Andres-Hernando A, Li N, Cicerchi C, et al. Protective role of fructokinase blockade in the pathogenesis of acute kidney injury in mice. Nat Commun 2017;8:14181.

66. Lanaspa MA, Ishimoto T, Cicerchi C, et al. Endogenous fructose production and fructokinase activation mediate renal injury in diabetic nephropathy. J Am Soc Nephrol 2014;25:2526-2538.

67. Roncal-Jimenez CA, Ishimoto T, Lanaspa MA, et al. Aging-associated renal disease in mice is fructokinase dependent. $\mathrm{Am} \mathrm{J}$ Physiol Renal Physiol 2016;311:F722-F730.

68. Lanaspa MA, Kuwabara M, Andres-Hernando A, et al. High salt intake causes leptin resistance and obesity in mice by stimulating endogenous fructose production and metabolism. Proc Natl Acad Sci U S A 2018;115:3138-3143. 
69. Mirtschink P, Krishnan J, Grimm F, et al. HIF-driven SF3B1 induces KHK-C to enforce fructolysis and heart disease. Nature 2015;522:444-449.

70. Hayward BE, Bonthron DT. Structure and alternative splicing of the ketohexokinase gene. Eur J Biochem 1998;257:85-91.

71. Diggle CP, Shires M, Leitch D, et al. Ketohexokinase: expression and localization of the principal fructose-metabolizing enzyme. J Histochem Cytochem 2009;57:763-774.

72. Asipu A, Hayward BE, O’Reilly J, Bonthron DT. Properties of normal and mutant recombinant human ketohexokinases and implications for the pathogenesis of essential fructosuria. Diabetes 2003;52:2426-2432.

73. Lecoultre V, Benoit R, Carrel G, et al. Fructose and glucose co-ingestion during prolonged exercise increases lactate and glucose fluxes and oxidation compared with an equimolar intake of glucose. Am J Clin Nutr 2010;92:1071-1079.

74. San-Millán I, Brooks GA. Reexamining cancer metabolism: lactate production for carcinogenesis could be the purpose and explanation of the Warburg effect. Carcinogenesis 2017;38:119-133.

75. Stincone A, Prigione A, Cramer T, et al. The return of metabolism: biochemistry and physiology of the pentose phosphate pathway. Biol Rev Camb Philos Soc 2015;90:927-963.

76. Lanaspa MA, Sanchez-Lozada LG, Cicerchi C, et al. Uric acid stimulates fructokinase and accelerates fructose metabolism in the development of fatty liver. PLoS One 2012;7:e47948.

77. Woods HF, Eggleston LV, Krebs HA. The cause of hepatic accumulation of fructose 1-phosphate on fructose loading. Biochem J 1970;119:501-510.

78. Lanaspa MA, Sanchez-Lozada LG, Choi YJ, et al. Uric acid induces hepatic steatosis by generation of mitochondrial oxidative stress: potential role in fructose-dependent and -independent fatty liver. J Biol Chem 2012;287:40732-40744.

79. Nakagawa T, Lanaspa MA, Millan IS, et al. Fructose contributes to the Warburg effect for cancer growth. Cancer Metab 2020;8:16.

80. Warburg O. On respiratory impairment in cancer cells. Science 1956;124:269-270.

81. Koppenol WH, Bounds PL, Dang CV. Otto Warburg's contributions to current concepts of cancer metabolism. Nat Rev Cancer 2011;11:325-337.

82. Chen Z, Liu M, Li L, Chen L. Involvement of the Warburg effect in non-tumor diseases processes. J Cell Physiol 2018;233:28392849.

83. Yang D, Wang MT, Tang Y, et al. Impairment of mitochondrial respiration in mouse fibroblasts by oncogenic H-RAS(Q61L). Cancer Biol Ther 2010;9:122-133.
84. Pfeiffer T, Schuster S, Bonhoeffer S. Cooperation and competition in the evolution of ATP-producing pathways. Science 2001;292:504-507.

85. Lunt SY, Vander Heiden MG. Aerobic glycolysis: meeting the metabolic requirements of cell proliferation. Annu Rev Cell Dev Biol 2011;27:441-464.

86. Rowe I, Chiaravalli M, Mannella V, et al. Defective glucose metabolism in polycystic kidney disease identifies a new therapeutic strategy. Nat Med 2013;19:488-493.

87. Chiaravalli M, Rowe I, Mannella V, et al. 2-Deoxy-d-glucose ameliorates PKD progression. J Am Soc Nephrol 2016;27:1958-1969.

88. Naudi A, Jove M, Ayala V, et al. Cellular dysfunction in diabetes as maladaptive response to mitochondrial oxidative stress. Exp Diabetes Res 2012;2012:696215.

89. Sharma K, Karl B, Mathew AV, et al. Metabolomics reveals signature of mitochondrial dysfunction in diabetic kidney disease. $J$ Am Soc Nephrol 2013;24:1901-1912.

90. Sas KM, Kayampilly P, Byun J, et al. Tissue-specific metabolic reprogramming drives nutrient flux in diabetic complications. JCI Insight 2016;1:e86976.

91. Ding H, Jiang L, Xu J, et al. Inhibiting aerobic glycolysis suppresses renal interstitial fibroblast activation and renal fibrosis. $\mathrm{Am} \mathrm{J}$ Physiol Renal Physiol 2017;313:F561-F575.

92. Werle M, Kreuzer J, Höfele J, et al. Metabolic control analysis of the Warburg-effect in proliferating vascular smooth muscle cells. J Biomed Sci 2005;12:827-834.

93. DeBerardinis RJ, Chandel NS. We need to talk about the Warburg effect. Nat Metab 2020;2:127-129.

94. Goraya N, Munoz-Maldonado Y, Simoni J, Wesson DE. Fruit and vegetable treatment of chronic kidney disease-related metabolic acidosis reduces cardiovascular risk better than sodium bicarbonate. Am J Nephrol 2019;49:438-448.

95. Rebholz CM, Crews DC, Grams ME, et al. DASH (dietary approaches to stop hypertension) diet and risk of subsequent kidney disease. Am J Kidney Dis 2016;68:853-861.

96. Nijveldt RJ, van Nood E, van Hoorn DE, Boelens PG, van Norren K, van Leeuwen PA. Flavonoids: a review of probable mechanisms of action and potential applications. Am J Clin Nutr 2001;74:418-425.

97. Berger L, Gerson CD, Yü TF. The effect of ascorbic acid on uric acid excretion with a commentary on the renal handling of ascorbic acid. Am J Med 1977;62:71-76.

98. Wu TK, Wei CW, Pan YR, et al. Vitamin C attenuates the toxic effect of aristolochic acid on renal tubular cells via decreasing oxidative stress-mediated cell death pathways. Mol Med Rep 
2015;12:6086-6092.

99. Reungjui S, Hu H, Mu W, et al. Thiazide-induced subtle renal injury not observed in states of equivalent hypokalemia. Kidney Int 2007;72:1483-1492.

100. Nakagawa T, Lanaspa MA, Johnson RJ. The effects of fruit consumption in patients with hyperuricaemia or gout. Rheumatology (Oxford) 2019;58:1133-1141.

101. Du H, Li L, Bennett D, et al. Fresh fruit consumption and major cardiovascular disease in China. N Engl J Med 2016;374:13321343.

102. Zheng J, Yang B, Tuomasjukka S, Ou S, Kallio H. Effects of latitude and weather conditions on contents of sugars, fruit acids, and ascorbic acid in black currant (Ribes nigrum L.) juice. $J$ Agric Food Chem 2009;57:2977-2987.

103. Nagy S. Vitamin C contents of citrus fruit and their products: a review. J Agric Food Chem 1980;28:8-18. 